\title{
Characterisation of normed linear spaces with Mazur's intersection property
}

\section{J.R. Giles, D.A. Gregory, and Brailey Sims}

\begin{abstract}
Normed linear spaces possessing the euclidean space property that every bounded closed convex set is an intersection of closed balls, are characterised as those with dual ball having weak * denting points norm dense in the unit sphere. A characterisation of Banach spaces whose duals have a corresponding intersection property is established. The question of the density of the strongly exposed points of the ball is examined for spaces with such properties.
\end{abstract}

It was Mazur [7] who drew attention to the euclidean space property $(I):$

every bounded closed convex set can be represented as an intersection of closed balls;

and he began the investigation to determine those normed linear spaces which possess this property. Phelps [9] continued this investigation, characterising finite dimensional spaces with property $(I)$. Recently, Sullivan [12] has given a characterisation of smooth spaces with property $(I)$.

Received 10 November 1977 . This paper was written while the authors were visiting scholars at the University of Washington in 1977 . They wish to thank the University of Washington for its hospitality and to express their appreciation at being able to participate in the Rainwater Seminar led by Professor R.R. Phelps. They wish to acknowledge their discussions with Mark Smith and Francis Sullivan. 
We develop Sullivan's key idea, and in Theorem 2.I characterise normed linear spaces with property $(I)$ as those with dual ball having weak * denting points norm dense in the unit sphere. In this theorem we actually give four equivalents for property $(I)$, two of which had been given by Phelps as necessary conditions, [9, p. 979], and two others related to Sullivan's approach.

For a dual space the appropriate intersection property which corresponds to property $(I)$ is property (weak * I):

every bounded weak * closed convex set can be represented as an intersection of closed balls.

In Theorem 3.1 we characterise Banach spaces with property (weak * I) on the dual as those with ball having denting points norm dense in the unit sphere.

Phelps proved that a normed linear space has property $(I)$ if the weak * strongly exposed points of the dual ball are norm dense in the unit sphere, [9, p. 977], and he raised the question of the necessity of this condition for property $(I)$. The corresponding question for property (weak * I) concerns the norm density of the strongly exposed points of the ball in the unit sphere. We show, in particular, that when a Banach space has both property $(I)$ and property (weak * $*$ ) on the dual, then both these density properties hold.

\section{Preliminaries}

We consider real normed linear spaces. Given a normed linear space $X$, and $y \in X$ and $r>0$, we denote by $B[y ; r]$ the closed ball $\{x \in X:\|x-y\| \leq r\}$. We denote by $B(X)$ the closed unit bazl $\{x \in X:\|x\| \leq I\}$ and by $S(X)$ the unit sphere $\{x \in X:\|x\|=1\}$. For $x \in S(X)$, we denote by $D(x)$ the set $\left\{f \in S\left(X^{*}\right): f(x)=1\right\}$, and for $f \in S\left(X^{*}\right)$, we denote by $D^{-1}(f)$ the set $\{x \in S(X): f(x)=1\}$. (For every $x \in S(X), D(x)$ is non-empty, but for some $f \in S\left(X^{*}\right), D^{-1}(f)$ may be empty.) We say that $X$ is smooth at $x \in S(X)$, if $D(x)$ is a single point set. The set valued mapping $x \mapsto D(x)$ of $S(X)$ into subsets of $S\left(X^{*}\right)$ is called the duality mapping on $X$. The inverse duality mapping on $X^{*}$ is the set valued mapping $f \mapsto D^{-1}(f)$ of $D(S(X))$ 
into subsets of $S(X)$. A mapping $x \mapsto f_{x}$ of $S(X)$ into $S\left(X^{*}\right)$, where $f_{x} \in D(x)$, is called a support mapping on $X$.

A slice of the ball $B(X)$ determined by $f \in S\left(X^{*}\right)$ is a set of the form $S(B(X), f, \delta) \equiv\{x \in B(X): f(x)>1-\delta\}$ for some $0<\delta<1$. A slice of the ball $B\left(X^{*}\right)$ determined by $x \in S(X)$ is sometimes called a weak * slice of $B\left(X^{*}\right)$. We say that $x \in S(X)$ is a denting point of $B(X)$ if, for every $\varepsilon>0, x$ is contained in a slice of $B(X)$ of diameter less than $\varepsilon$. We say that $f \in S\left(X^{*}\right)$ is a weak * denting point of $B\left(X^{*}\right)$ if, for every $\varepsilon>0, f$ is contained in a weak * slice of $B\left(X^{*}\right)$ of diameter less than $\varepsilon$. We say that $x \in S(X)$ is a strongly exposed point of $B(X)$, if there exists an $f \in S\left(X^{*}\right)$ such that, for every $\varepsilon>0, f$ determines a slice of $B(X)$ containing $x$ and of diameter less than $\varepsilon$. We say that $f \in S\left(X^{*}\right)$ is a weak * strongly exposed point of $B\left(X^{*}\right)$, if there exists an $x \in S(X)$ such that, for every $\varepsilon>0, x$ determines a slice of $B\left(X^{*}\right)$ containing $f$ and of diameter less than $\varepsilon$. A strongly exposed point is a denting point, but the converse is not true even in finite dimensional spaces.

We have the following elementary but useful property for slices.

LEMMA 1.1. In a normed linear space $X$, consider $x \in S(X)$. For any slice determined by an $f \in S\left(X^{*}\right)$ and containing $x$ there exists an $\varepsilon>0$ such that for all $g \in S\left(X^{*}\right)$, where $\|f-g\|<\varepsilon$, there exists a slice determined by $g$ which contains $x$ and is contained in the slice determined by $f$.

Proof. Suppose $x \in S(B(X), f, \delta)$. Choose $\varepsilon<\frac{1}{2}(f(x)-1+\delta)$. Then for $\|f-g\|<\varepsilon$ we have $g(x) \geq f(x)-\varepsilon>1-(\delta-\varepsilon)$; so $x \in S(B(X), g, \delta-\varepsilon)$. Also for all $y \in S(B(X), g, \delta-\varepsilon)$ we have $f(y) \geq g(y)-\varepsilon>1-\delta$; so $y \in S(B(X), f, \delta)$.

From the Bishop-Phelps Theorem we make an immediate deduction.

COROLLARY 1.1. For a Banach space $X$, consider $x \in S(X)$. For any slice determined by an $f \in S\left(X^{*}\right)$ and containing $x$ there exists a slice determined by a $g \in D(S(X))$ which contains $x$ and is contained in the slice determined by $f$.

As we might guess, conditions for property $(I)$ must involve some 
smoothness condition on the unit sphere, and the problem has been to find the right condition for a characterisation. Both Mazur and Phelps concentrated on conditions involving strong differentiability of the norm. The norm of a normed space $X$ is said to be strongly differentiable (Fréchet differentiable) at $x \in S(X)$, if for all $y \in S(X)$ and real $\lambda$,

$$
\lim _{\lambda \rightarrow 0} \frac{\|x+\lambda y\|-\|x\|}{\lambda}
$$

exists and is approached uniformly for all $y \in S(X)$. If the norm is strongly differentiable at $x \in S(X)$, then $X$ is smooth at $x$. The norm of $X$ is strongly differentiable at $x \in S(X)$, if and only if $f_{x} \in D(x)$ is a weak * strongly exposed point of $B\left(X^{*}\right)$ by $x$. The norm of $X^{*}$ is strongly differentiable at $f \in S\left(X^{*}\right)$, if and only if there exists an $x \in S(X)$ such that $f \in D(x)$ and $x$ is a strongly exposed point of $B^{\prime}(X)$ by $f[10$, Theorem 1$]$.

Sullivan, instead of concentrating on the set of points of strong differentiability of the norm in $S(X)$, considered more general sets. For given $\varepsilon>0$, consider the set $M_{\varepsilon}(X)$ consisting of points $x \in S(X)$ such that for some $\delta(\varepsilon, x)>0$,

$$
\sup _{\substack{0<\lambda<\delta \\ y \in S(X)}} \frac{\|x+\lambda y\|+\|x-\lambda y\|-2}{\lambda}<\varepsilon .
$$

We note that the set of points of $S(X)$ where the norm of $X$ is strongly differentiable is precisely the set $\bigcap_{\varepsilon>0} M_{\varepsilon}(X)$. Sullivan also established an important link with strong differentiability of the norm in the following result [12, $\$ 3$, Corollary 6]. Another link is given in Corollary 2.2 below.

THEOREM 1.1. For a Banach space $X$, if $M_{E}(X)=S(X)$ for some $0<\varepsilon<1$ then $X$ is an Asplund space (that is, every equivalent norm for $X$ is strongly differentiable on a norm dense subset of $S(X) \quad[8$, p. 749]).

However, the $M_{\varepsilon}(X)$ sets in $S(X)$ also relate to the weak * denting points of $B\left(X^{*}\right)$ and the $M_{\varepsilon}\left(X^{*}\right)$ sets in $S\left(X^{*}\right)$ relate to the 
denting points of $B(X)$.

LEMMA 1.2. (i) For a normed linear space $X$, consider $A \subseteq S(X)$ where $D(A)$ is norm dense in $S\left(X^{*}\right)$. Then $\bar{A}$ contains the denting points of $B(X)$.

(ii) For a normed linear space $X$, consider $A \subseteq S\left(X^{*}\right)$ where $D^{-1}(A)$ is norm dense in $S(X)$. Then $\bar{A}$ contains the weak * denting points of $B\left(X^{*}\right)$.

Proof. (i) Consider $x \in S(X)$ a denting point of $B(X)$. Since $D(A)$ is norm dense in $S\left(X^{*}\right)$, we have by Lemma 1.1 that for each $\varepsilon>0$ there exists a $y \in S(X) \cap A$ and $f_{y} \in D(y)$ such that $f_{y}$ determines a slice of $B(X)$ which contains $x$ and has diameter less than $\varepsilon$. As $y \in A$ belongs to this slice, $\|x-y\|<\varepsilon$, and so $x \in \bar{A}$.

(ii) This proof follows similarly, applying Lemma 1.1 to $X^{*}$.

In particular, we can make the following deduction.

COROLLARY 1.2. (i) For a normed linear space $X$, if for every $\varepsilon>0, D\left(M_{\varepsilon}(X)\right)$ is norm dense in $S\left(X^{*}\right)$, then $\underset{\varepsilon>0}{\cap} \overline{M_{\varepsilon}(X)}$ contains the denting points of $B(X)$.

(ii) For a normed linear space $X$, if for every $\varepsilon>0$, $D^{-1}\left(M_{\varepsilon}\left(X^{*}\right)\right)$ is norm dense in $S(X)$, then $\bigcap_{\varepsilon>0} \overline{M_{\varepsilon}\left(X^{*}\right)}$ contains the weak * denting points of $B\left(X^{*}\right)$.

\section{Characterisation of spaces with property (I)}

We approach our theorem by characterising points in $M_{\varepsilon}(X)$ sets. Lemma 2.1 and the corresponding Lemma 3.1 generalise results given initially by Śmulian, for points of strong differentiability of the norm [11, p. 645].

LEMMA 2.1. For a normed linear space $X$, given $\varepsilon>0$, the following statements are equivalent:

$$
\begin{aligned}
& \text { (i) } x \in M_{\varepsilon}(X) ; \\
& \text { (ii) } x \text { determines a slice of } B\left(X^{*}\right) \text { of diameter less than } \varepsilon \text {; }
\end{aligned}
$$


(iii) for all sequences $f_{n} \in S\left(X^{*}\right)$ such that $f_{n}(x) \rightarrow 1$,

$$
\lim \sup \left\|f_{n}-f_{m}\right\|<\varepsilon \text {; }
$$

(iv) for all sequences $x_{n} \in S(X)$ where $x_{n} \rightarrow x$, and all

$$
f_{x_{n}} \in D\left(x_{n}\right) \text {, }
$$

$$
\lim \sup \left\|f_{x_{n}}-f_{x_{m}}\right\|<\varepsilon \text {. }
$$

Proof. (i) $\Rightarrow$ (ii). Suppose that diam $S\left(B\left(X^{*}\right), x, \delta\right) \geq \varepsilon$ for all $0<\delta<1$. Then there exist $f_{n}, g_{n} \in B\left(X^{*}\right)$ such that

$$
f_{n}(x)>1-1 / n^{2}, g_{n}(x)>1-1 / n^{2} \text {, and }\left\|f_{n}-g_{n}\right\|>\varepsilon-1 / n \text {. }
$$

Choose $y_{n} \in S(X)$ such that $\left(f_{n}-g_{n}\right)\left(y_{n}\right)>\varepsilon-1 / n$.

Then

$$
\begin{aligned}
\left\|x+\frac{1}{n} y_{n}\right\|+\left\|x-\frac{1}{n} y_{n}\right\| & \geq f_{n}\left(x+\frac{1}{n} y_{n}\right)+g_{n}\left(x-\frac{1}{n} y_{n}\right) \\
& >2-\frac{2}{n^{2}}+\frac{1}{n}\left(f_{n}-g_{n}\right)\left(y_{n}\right) \\
& >2-\frac{3}{n^{2}}+\frac{\varepsilon}{n} .
\end{aligned}
$$

Therefore,

$$
\frac{\left\|x+(1 / n) y_{n}\right\|+\left\|x-(1 / n) y_{n}\right\|-2}{1 / n}>\varepsilon-\frac{3}{n} \text { for all } n \text {. }
$$

(ii) $\Rightarrow$ (iii). If there exists a $\delta>0$ such that

diam $S\left(B\left(X^{*}\right), x, \delta\right)<c$, then $f_{n}^{*} \in S\left(B\left(X^{*}\right), x, \delta\right)$ for $n$ sufficiently large.

$$
\begin{aligned}
& \quad \text { (iii) } \Rightarrow \text { (iv). }\left|f_{x_{n}}(x)-1\right| \leq\left|f_{x_{n}}\left(x-x_{n}\right)\right| \leq\left\|x-x_{n}\right\| \text {, so that } \\
& f_{x_{n}}(x)+1 . \\
& \quad(i v) \Rightarrow \text { (i). For any given } y \in S(X) \text { and } \lambda_{n}+0+\text {, and for any }
\end{aligned}
$$




$$
f_{n} \in D\left(\frac{x+\lambda n^{y}}{\left\|x+\lambda_{n} y\right\|}\right) \text { and } g_{n} \in D\left(\frac{x-\lambda n^{y}}{\left\|x-\lambda_{n} y\right\|}\right) \text {, }
$$

we have

$$
\begin{aligned}
\frac{\| x+\lambda n_{n}^{y\|+\| x-\lambda} n^{y \|-2}}{\lambda_{n}} & \leq f_{n}(y)-g_{n}(y) \\
& \leq\left\|f_{n}-g_{n}\right\|
\end{aligned}
$$

$[5 ; \mathrm{p}: 108]$. But

$$
\begin{aligned}
\left\|\frac{x+\lambda n^{y}}{\left\|x+\lambda n^{y}\right\|}-x\right\| & \leq\left\|x+\lambda_{n} y-\frac{x+\lambda_{n} y}{\left\|x+\lambda_{n} y\right\|}\right\|+\left\|\lambda_{n} y\right\| \\
& =\left|\left\|x+\lambda_{n} y\right\|-\|x\|\right|+\left|\lambda_{n}\right| \leq 2\left|\lambda_{n}\right| .
\end{aligned}
$$

So there exists a $0<\delta(\varepsilon, x)<1$, and, from $(i v)$,

$$
\sup _{\substack{0<\lambda_{n}<\delta \\ y \in S(X)}} \frac{\left\|x+\lambda_{n} y\right\|+\left\|x-\lambda_{n} y\right\|-2}{\lambda_{n}}<\varepsilon .
$$

The following consequences are immediate from this lemma.

COROLLARY 2.1. For a normed linear space $X$, given $\varepsilon>0$, the set $M_{\varepsilon}(X)$ is open in $S(X)$.

Proof. Consider $x \in M_{\varepsilon}(X)$. By Lemma 2.1 (ii), $x$ determines a slice of $B\left(X^{*}\right)$ of diameter less than $\varepsilon$. By Lemma 1.1 applied to $X^{*}$, if $y \in S(X)$ and $\|x-y\|$ is sufficiently small, $y$ also determines a slice of $B\left(X^{*}\right)$ of diameter less than $\varepsilon$, so that by Lemma 2.1 (i), $y \in M_{\varepsilon}(X)$.

Corollary 2.1 enables us to establish another link with strong differentiability of the norm.

COROLLARY 2.2. For a Banach space $X$, if $M_{\varepsilon}(X)$ is norm dense in $S(X)$ for every $0<\varepsilon<1$, then the norm of $X$ is strongly differentiable on a norm dense $G_{\delta}$ subset of $S(X)$.

Proof. By the Baire Category Theorem, $\bigcap_{\varepsilon>0} M_{\varepsilon}(X)$ is norm dense in 
$S(X)$, but $\prod_{\varepsilon>0} M_{\varepsilon}(X)$ is the subset of $S(X)$ where the norm is strongly differentiable.

The following reflexivity result is a special case of a lemma of Sullivan [12, Lemma, §3.3], but the proof is somewhat simpler.

COROLLARY 2.3. If for a Banach space $X$ there exists some $0<\varepsilon<1$ such that $D\left(M_{\varepsilon}\left(X^{*}\right)\right)$ is norm dense in $S\left(X^{*}\right)$, then $X$ is reflexive.

Proof. Since $\varepsilon<1$, it is sufficient to show that each $F \in D\left(M_{\varepsilon}\left(X^{*}\right)\right)$ is within $\varepsilon$ distance of $\hat{X}$. Since $B(\hat{X})$ is weak * dense in $B\left(X^{*}\right)$ each weak * slice of $B\left(X^{*}\right)$ containing $F$ contains some element of $B(\hat{X})$. But by Lemma $2.1(i i), F$ is in such a weak * slice of diameter less than $\varepsilon$.

In particular, using the Bishop-Phelps Theorem we can make the following deduction from Corollary 2.3. This generalises the well known result that a Banach space $X$ is reflexive if the norm of $X^{*}$ is strongly differentiable on $S\left(X^{*}\right)$.

COROLLARY 2.4. If for a Banach space $X$ there exists some $0<\varepsilon<1$ such that $M_{\varepsilon}\left(X^{*}\right)=S\left(X^{*}\right)$, then $X$ is reflexive.

We now give a characterisation of functionals which satisfy a certain separation property. This is a local property which globally makes up property $(I)$.

LEMMA 2.2. For a normed linear space $X$, given $f \in S\left(X^{*}\right)$, the following statements are equivalent:

(i) $f \in \bigcap_{\varepsilon>0} \overline{D\left(M_{\varepsilon}(X)\right)}$;

(ii) for every bounded set $C$ with inf $f(C)>0$ there exists a closed ball containing $C$ which does not contain 0 ;

(iii) given $0<\varepsilon<1$ there exists an $x \in S(X)$ and $a$ $\delta(\varepsilon)<0$ such that $D(y) \subseteq B(f ; \varepsilon)$ for alz $y \in S(X) \cap B(x ; \delta)$.

Proof. (i) $\Rightarrow(i i)$. Let $C$ be a bounded set with inf $f(c)>0$. 
There exists a $k>0$ such that $C \subseteq B[0 ; k]$. Choose $\varepsilon \equiv \frac{1}{3}$ inf $f(c)$. Since $f \in \overline{D\left(M_{\varepsilon / k}(X)\right)}$ there exists an $x \in M_{\varepsilon / k}(X)$ and an $f_{x} \in D(x)$ such that $\left\|f-f_{x}\right\|<\varepsilon / k$. Consider the sequence of closed balls $B_{n} \equiv B[n \varepsilon x ;(n-1) \varepsilon]$. We show that there exists an $n_{0}$ such that $C \subseteq B_{n_{0}}$. Suppose otherwise, that for every $n$ there exists an $x_{n} \in C \backslash B_{n}$. Write $y_{n} \equiv \frac{x_{n}}{n \varepsilon} ;$ then since $\left\{x_{n}\right\}$ is bounded, $y_{n} \rightarrow 0$. But

$$
\begin{aligned}
\frac{\left\|x+y_{n}\right\|+\left\|x-y_{n}\right\|-2}{\left\|y_{n}\right\|} & =\frac{\left\|x+y_{n}\right\|-\|x\|}{\left\|y_{n}\right\|}+\frac{\left\|x_{n}-n \varepsilon x\right\|-n \varepsilon}{\left\|x_{n}\right\|} \\
& \geq f_{x}\left(\frac{y_{n}}{\left\|y_{n}\right\|}\right)+\frac{(n-1) \varepsilon-n \varepsilon}{\left\|x_{n}\right\|}[5, \text { p. 108], } \\
& \geq \frac{f\left(x_{n}\right)-\varepsilon}{\left\|x_{n}\right\|}-\left\|f-f_{x}\right\|>\varepsilon / k,
\end{aligned}
$$

and this contradicts $x \in M_{\varepsilon / k}(X)$.

$$
\text { (ii) } \Rightarrow \text { (iii). Consider } D \equiv B(X) \cap f^{-1}(0) \text {, and let } u \in S(X) \text { be }
$$

such that $f(u)>\varepsilon$. Write $u^{\prime} \equiv \frac{\varepsilon}{2} u$. Then $f\left(D+u^{\prime}\right)=\frac{\varepsilon^{2}}{2}>0$ and $0 \notin D+u^{\prime}$. So there exists a closed ball containing $D+u^{\prime}$ and not containing 0 . Therefore there exists a closed ball containing $D$ and not containing $u^{\prime}$. The proof now follows identically that of Lemma 4.1 of Phelps [9, p. 979].

(iii) $\Rightarrow$ (i). Given $\varepsilon>0$ and $0<\eta<\varepsilon / 2$, there exists an $x \in S(X)$ and a $\delta(\eta)>0$ such that $D(y) \subseteq B(f ; \eta)$ for all $y \in S(X) \cap B(x ; \delta)$. In particular $D(x) \subseteq B(f ; \eta)$, and for all $x_{n} \in S(X)$ where $x_{n} \rightarrow x$ we have that $\lim \sup \left\|f_{x_{n}}-f_{x_{m}}\right\| \leq 2 n<\varepsilon$ for all $f_{x_{n}} \in D\left(x_{n}\right)$. But by Lemma 2.1 (iv) this implies that $x \in M_{\varepsilon}(X)$. So $\left\|f-f_{x}\right\|<\eta$, where $f_{x} \in D\left(M_{\varepsilon}(X)\right)$.

The localised form of one of Mazur's results [7, p. 128] follows 
directly from Lemma 2.2 (ii).

COROLLARY 2.5. For a normed linear space $X$, let $f \in S\left(X^{*}\right)$ have any of the properties of Lemma 2.2. Then, for any bounded sequence $\left\{x_{n}\right\}$ such that every closed ball containing a subsequence also contains $x$, we have that $f\left(x_{n}\right) \rightarrow f(x)$.

We say that the duality mapping $x \mapsto D(x)$ on $X$ is quasi-continuous if, given $f \in S\left(X^{*}\right)$ and $0<\varepsilon<1$, there exists an $x \in S(X)$ and a $\delta(\varepsilon, f)>0$ such that $D(y) \subseteq B(f ; \varepsilon)$ for all $y \in S(X) \cap B(x ; \delta)$.

THEOREM 2.1. FOr a normed linear space $X$, the following statements are equivalent:

(i) $X$ has property (I);

(ii) the duality mapping on $X$ is quasi-continuous;

(iii) every support mapping on $X$ maps norm dense sets in $S(X)$ to norm dense sets in $S\left(X^{*}\right)$;

(iv) for every $\varepsilon>0, D\left(M_{\varepsilon}(X)\right)$ is norm dense in $S\left(X^{*}\right)$;

(v) the weak * denting points of $B\left(X^{*}\right)$ are norm dense in $S\left(X^{*}\right)$.

Proof. $(i) \Rightarrow(i i)$. If the duality mapping is not quasi-continuous, there exists an $f \in S\left(X^{*}\right)$ which does not obey the property given in Lemma 2.2 ( $i$ ii). So by Lemma 2.2 ( $i i$ ) there exists a bounded closed convex set $C$ with inf $f(C)>0$ and every closed ball containing $C$ also contains 0 . So then $C$ cannot be represented as an intersection of closed balls. (This is Phelps' Lemma 4.1 [9, p. 979].)

(ii) $\Rightarrow$ (iii). It follows directly from the definition of quasicontinuity that for any support mapping $\phi$ on $X$, if $A$ is norm dense in $S(X)$, then $\phi(A)$ is norm dense in $S\left(X^{*}\right)$. (This is Phelps' Corollary $4.2[9$, p. 980].)

$(i i i) \Rightarrow(i i)$. Suppose that the duality mapping is not quasicontinuous. Then there exists an $f \in S\left(X^{*}\right)$ and an $r>0$ such that, for every $x \in S(X)$ and $\varepsilon>0$, there exists a $y \in B(x ; \varepsilon)$, where $D(y) \nsubseteq B(f ; \boldsymbol{r})$. So there exists a norm dense set $A$ in $S(X)$ and a support mapping $\phi$, where $\phi(A) \cap B(f ; r)=\varnothing$. 
(ii) $\Rightarrow$ (iv). If the duality mapping is quasi-continuous, it follows from Lemma $2.2(i)$ that $S\left(X^{*}\right)=\bigcap_{\varepsilon>0} \overline{D\left(M_{\varepsilon}(X)\right)}$.

$(i v) \Rightarrow(v)$. For given $\varepsilon>0$, we have by Lemma 2.1 (ii) that $D\left(M_{\varepsilon}(X)\right)$ is contained in the set $D_{\varepsilon}$, the union of points in $S\left(X^{*}\right)$ which are weak * interior to weak * slices of $B\left(X^{*}\right)$ of diameter less than $\varepsilon$. So for each $\varepsilon>0, D_{\varepsilon}$ is norm open and norm dense in $S\left(X^{*}\right)$. By the Baire Category Theorem $\bigcap_{\varepsilon>0} D_{\varepsilon}$ is norm dense in $S\left(X^{*}\right)$; but these are precisely the weak * denting points of $B\left(X^{*}\right)$.

$(v) \Rightarrow(i v)$. Consider $f \in S\left(X^{*}\right)$, a weak * denting point of $B\left(X^{*}\right)$. Given $\varepsilon>0$ and $0<n<\varepsilon$, there exists an $x \in S(X)$ such that $f$ belongs to a slice determined by $x$ of diameter less than $\eta$. Now $D(x)$ is contained in this slice. Therefore $\left\|f_{x}-f\right\|<\eta$ for any $f_{x} \in D(x)$, and by Lemma 2.1 (ii), $x \in M_{\eta}(X) \subseteq M_{\varepsilon}(X)$. So $f \in \overline{D\left(M_{\varepsilon}(X)\right)}$.

$(i v) \Rightarrow(i)$. Consider a bounded closed convex set $C$ and a point $y \in X \backslash C$. We may assume that $y=0$. By the Separation Theorem there exists a continuous linear functional $f$ such that inf $f(C)>0$. Now $f \in \bigcap_{\varepsilon>0} \overline{D\left(M_{\varepsilon}(X)\right)}$, so by Lemma 2.2 (ii) there exists a closed ball containing $C$ which does not contain 0 .

A weak * denting point is an extreme point. In a finite dimensional space an extreme point is a weak * denting point, so Phelps' result [9, p. 980] is immediate.

COROLLARY 2.6. A finite dimensional normed linear space $X$ has property (I), if and only if the set of extreme points of $B\left(X^{*}\right)$ is dense in $S\left(X^{*}\right)$.

From Corollary 2.3 the following result is immediate.

COROLLARY 2.7. A Banach space $X$ whose dual $X^{*}$ has property (I) is reflexive.

Using the fact that $\bigcap_{\varepsilon>0} \overline{D\left(M_{\varepsilon}(X)\right)}$ contains the weak * denting points of $B\left(X^{*}\right)$ and that for a reflexive space $B\left(X^{*}\right)$ is the closed convex hull of its denting points [4, p. 25], we can deduce the following 
extension of Mazur's result given locally in Corollary 2.5.

COROLLARY 2.8. For a reflexive Banach space with property $(I)$, a bounded sequence $\left\{x_{n}\right\}$ converges weakly to $x$, if and only if every closed ball containing a subsequence also contains $x$.

In his paper [9, p. 982], Phelps asked whether $X$ having property (I) implies that the set of weak * strongly exposed points of $B\left(X^{*}\right)$ is norm dense in $S\left(X^{*}\right)$. In the light of Theorem 2.1 this question takes the following form.

PROBLEM 2.1. If $X$ has the set of weak * denting points of $B\left(X^{*}\right)$ norm dense in $S\left(X^{*}\right)$, is the set of weak * strongly exposed points of $B\left(X^{*}\right)$ norm dense in $S\left(X^{*}\right)$ ?

If the norm of $X$ is strongly differentiable on a norm dense set in $S(X)$, then from Theorem 2.1 ( $i i i$ ) we have an affirmative answer to our question. Asplund spaces satisfy this condition. So then all reflexive Banach spaces and all separable Banach spaces with property $(I$ ) satisfy this condition. This leads us to ask the further question:

PROBLEM 2.2. Is every Banach space with property $(I)$ an Asplund space?

We point out that if $X$ has the property that every point of $D(S(X))$ is a weak * denting point of $B\left(X^{*}\right)$, then the norm of $X$ is strongly differentiable on $S(X)$. So from Theorem 2.1 (iii) the set $D(S(X)$ ) of weak * strongly exposed points of $B\left(X^{*}\right)$ is norm dense in $S\left(X^{*}\right)$, and if $X$ is a Banach space, then from Theorem 1.1, $X$ is an Asplund space.

\section{Characterisation of spaces with property (weak * I)}

By developing Lemmas 3.1 and 3.2 for the dual space similar to Lemmas 2.1 and 2.2 we are able to establish a characterisation theorem for dual spaces with property (weak * I) similar to that given in Theorem 2.1 for spaces with property $(I)$.

LEMMA 3.1. For a Banach space $X$, given $\varepsilon>0$, the following statements are equivalent:

(i) $f \in M_{\varepsilon}\left(X^{*}\right)$;

(ii) $f$ determines a stice of $B(X)$ of diameter less than $\varepsilon$; 
(iii) for all sequences $x_{n} \in S(X)$ such that $f\left(x_{n}\right) \rightarrow 1$, lim sup $\left\|x_{n}-x_{m}\right\|<\varepsilon$;

(iv) for all sequences $x_{n} \in S(X)$ and all $f_{x_{n}} \in D\left(x_{n}\right)$, where $f_{x_{n}}+f, \quad \lim \sup \left\|x_{n}-x_{m}\right\|<\varepsilon$.

Proof. (i) $\Rightarrow$ (ii) follows from $(i) \Rightarrow$ (ii) in Lemma 2.1.

(ii) $\Rightarrow$ (iii) and (iii) $\Rightarrow$ (iv) follow as in Lemme 2.1.

$(i v) \Rightarrow(i i i)$. From the Bollobás estimates for the Bishop-Phelps Theorem [1] we have that for every sequence $x_{n} \in S(X)$ such that $f\left(x_{n}\right) \rightarrow 1$, there exists a sequence $y_{n} \in S(X)$ and $f_{y_{n}} \in D\left(y_{n}\right)$ such that $\left\|x_{n}-y_{n}\right\| \rightarrow 0$ and $\left\|f-f_{y_{n}}\right\| \rightarrow 0$.

(iii) $\Rightarrow$ (i). Suppose that $f \notin M_{E}\left(X^{*}\right)$; then from Lemma 2.1 (iii) there exists a sequence $F_{n} \in S\left(X^{* *}\right)$ such that $F_{n}(f) \rightarrow 1$, but $\left\|F_{n}-F_{m}\right\| \geq \varepsilon$ for all $m, n$. So for each $n$ there exists $f_{n} \in S\left(X^{*}\right)$ such that $\left|\left(F_{n}-F_{n+1}\right)\left(f_{n}\right)\right| \geq \varepsilon-1 / n$. Since $B(\hat{X})$ is weak * dense in $B\left(X^{* *}\right)$, there exists a sequence $x_{n} \in S(X)$ such that

$$
\begin{gathered}
\left|\left(F_{n}-\hat{x}_{n}\right)\left(f_{n}\right)\right|<1 / n, \\
\left|\left(F_{n}-\hat{x}_{n}\right)\left(f_{n-1}\right)\right|<1 / n,
\end{gathered}
$$

and

$$
\left|\left(E_{n}-\hat{x}_{n}\right)(f)\right|<1 / n
$$

Then $f\left(x_{n}\right) \rightarrow 1 ;$ but

$$
\begin{aligned}
\left\|x_{n}-x_{n+1}\right\| & \geq\left|\left(\hat{x}_{n}-\hat{x}_{n+1}\right)\left(f_{n}\right)\right| \\
& \geq\left|\left(F_{n}-F_{n+1}\right)\left(f_{n}\right)\right|-\left|\left(\hat{x}_{n}-F_{n}\right)\left(f_{n}\right)\right|-\left|\left(\hat{x}_{n+1}-F_{n+1}\right)\left(f_{n}\right)\right| \\
& >\varepsilon-3 / n .
\end{aligned}
$$

So $\lim \sup \left\|x_{n}-x_{m}\right\| \geq \varepsilon$.

From Lemmas 2.1 and 3.1 we make the following deduction. 
COROLLARY 3.1. For a normed linear space $X$, if $x \in M_{\varepsilon}(X)$, then $\hat{x} \in M_{\varepsilon}\left(X^{* *}\right)$.

LEMMA 3.2. For a Banach space $X$, given $x \in S(X)$, the following statements are equivalent:

(i) $x \in \bigcap_{\varepsilon>0} \overline{D^{-1}\left(M_{\varepsilon}\left(X^{*}\right)\right)}$;

(ii) for every bounded set $C$ in $X^{*}$ with inf $\hat{x}(C)>0$, there exists a closed dual ball containing $C$ which does not contain 0 ;

(iii) given $0<\varepsilon<1$ there exists an $f \in D(S(X))$ and $a$ $\delta(\varepsilon)>0$ such that $D^{-1}(g) \subseteq B(x ; \varepsilon)$ for azz $g \in D(S(X)) \cap B(f ; \delta)$.

Proof. (i) $\Rightarrow(i i)$. Given $\varepsilon>0, D^{-1}\left(M_{\varepsilon}\left(X^{*}\right)\right) \subseteq D\left(M_{\varepsilon}\left(X^{*}\right)\right)$; so $x \in \bigcap_{\varepsilon>0} \overline{D\left(M_{\varepsilon}\left(X^{*}\right)\right]}$, and the result follows from Lemma 2.2 (ii).

$(i i) \Rightarrow(i i i)$. Given $\varepsilon>0$, we have from Lemma 2.2 ( $i i i$ ), that there exists an $f^{\prime} \in S\left(X^{*}\right)$ and a $\delta^{\prime}(\varepsilon)>0$ such that $D(g) \subseteq B(\hat{x} ; \varepsilon)$ for all $g \in S\left(X^{*}\right) \cap B\left(f^{\prime} ; \delta^{\prime}\right)$. But by the Bishop-Phelps Theorem there exists an $f \in D(S(X))$ and a $0<\delta<\delta^{\prime}$ such that $B(f ; \delta) \subseteq B\left(f^{\prime} ; \delta^{\prime}\right)$. So $D^{-1}(g) \subseteq B(x ; \varepsilon)$ for all $g \in D(S(X)) \cap B(f ; \delta)$.

(iii) $\Rightarrow$ (i). Given $\varepsilon>0$ and $0<\eta<\varepsilon / 2$, there exists an $f \in D(S(X))$ and a $\delta(n)>0$ such that $D^{-1}(f) \subseteq B(x ; n)$ for all $g \in D(S(X)) \cap B(f ; \delta)$. In particular, $D^{-1}(f) \subseteq B(x ; n)$ and for all $x_{n} \in S(X)$ and $f_{x_{n}} \in D\left(x_{n}\right)$, where $f_{x_{n}} \rightarrow f$, we have that lim $\sup \left\|x_{n}-x_{m}\right\|<2 n<\varepsilon$. By Lemma 3.1 (iv) this implies that $f \in M_{\varepsilon}\left(X^{*}\right)$. So $\left\|x-x_{f}\right\|<\eta$ where $x_{f} \in D^{-1}\left(M_{\varepsilon}\left(X^{*}\right)\right)$.

The inverse duality mapping $f \mapsto D^{-1}(f)$ on $X^{*}$ is quasi-contiraous if, given $x \in S(X)$ and $0<\varepsilon<1$, there exists an $f \in D(S(X))$ and a $\delta(\varepsilon, x)>0$ such that $D^{-1}(g) \subseteq B(x ; \varepsilon)$ for all $g \in D(S(X)) \cap B(f ; \delta)$.

THEOREM 3.1. For a Banach space $X$, the following statements are 
equivalent:

(i) $X^{*}$ has property (weak *I) ;

(ii) the inverse duality mapping on $X^{*}$ is quasi-continuous;

(iii) every support mapping on $X^{*}$ which maps $D(S(X))$ into $S(X)$ has the property that it maps norm dense sets in $D(S(X))$ to norm dense sets in $S(X)$;

(iv) for every $\varepsilon>0, D^{-1}\left(M_{\varepsilon}\left(X^{*}\right)\right)$ is norm dense in $S(X)$;

(v) the denting points of $B(X)$ are norm dense in $S(X)$.

Proof. All the proofs follow a similar pattern to those given in Theorem 2.1, but using Lemmas 3.1 and 3.2. The proof $(v) \Rightarrow$ (iv) also uses Corollary 1.1.

Our characterisation Theorem 3.1 raises the question dual to that raised by Theorem 2.1 .

PROBLEM 3.1. If $X$ has the set of denting points of $B(X)$ norm dense in $S(X)$, is the set of strongly exposed points of $B(X)$ norm dense in $S(X)$ ?

In connection with the question dual to that raised in Problem 2.2 we make the following remark. A normed linear space $X$ is locally uniformly rotund, if for every $x \in S(X)$ and sequence $x_{n} \in S(X)$ such that $\left\|x_{n}+x\right\| \rightarrow 2$, we have $x_{n} \rightarrow x$. It follows that in a locally uniformly rotund space $X$, every point of $S(X)$ is a strongly exposed point of $B(X)$, and so, from Theorem $3.1(v), X^{*}$ has property (weak * $I$ ). Using this fact we note that a Banach space $X$ with property (weak $* I$ ) on $X^{*}$ is not necessarily weak * Asplund; that is, not every equivalent dual norm for $X^{*}$ need be strongly differentiable on a norm dense subset of $S\left(X^{*}\right)$ [2, p. 103]. The space $c_{0}$ can be equivalently renormed to be locally uniformly rotund, but $\tau_{1}$ is not weak * Asplund.

4. Spaces with property $(I)$ and property (weak * I) on the dual

We have drawn attention to two significant questions in Problems 2.1 and 3.1. It is of interest to examine their solutions for spaces which 
combine all the special features associated with property $(I)$ and property (weak *I) on the dual.

THEOREM 4.1. A Banach space $X$ has property $(I)$ and $X^{*}$ has property (weak * $I$ ), if and only if the set of strongly exposed points of $B(X)$ is norm dense in $S(X)$ and the set of weak * strongly exposed points of $B\left(X^{*}\right)$ is norm dense in $S\left(X^{*}\right)$.

Proof. Suppose that $X$ has property $(I)$ and $X^{*}$ has property (weak * $* I$ ). From Theorem $2.1(i v)$, for every $\varepsilon>0, D\left(M_{\varepsilon}(X)\right)$ is norm dense in $S\left(X^{*}\right)$ and from Theorem $3.1(v)$, the denting points of $B(X)$ are norm dense in $S(X)$. From Corollary 1.2 (i) we deduce that, for every $\varepsilon>0, M_{\varepsilon}(X)$ is norm dense in $S(X)$. But from Corollary 2.2 the norm is strongly differentiable on a norm dense subset of $S(X)$. Similarly, using Theorems $3.1(i v), 2.1(v)$, and Corollary 1.2 (ii), we have that the set of points where the norm of $X^{*}$ is strongly differentiable is norm dense in $S\left(X^{*}\right)$. Applying Theorems 2.1 (iii) and 3.1 ( $i i i$ ) we have our result.

Conversely, the proof follows directly from Theorems $2.1(v)$ and 3.1 (v).

There are many examples of the spaces of Theorem 4.1.

THEOREM 4.2. Any weakly compactly generated Banach space with weakly compactly generated dual can be equivalently renormed to have property (I) and property (weak *I) on the dual.

Proof. Such a space $X$ can be equivalently renormed so that both $X$ and $X^{*}$ are locally uniformly rotund [6, p. 185]. It follows that every point of $S(X)$ is a strongly exposed point of $B(X)$ and every point of $D(S(X))$ is a weak * strongly exposed point of $B\left(X^{*}\right)$. The result follows from the Bishop-Phelps Theorem and Theorem 4.1.

\section{Uniform conditions}

It seems reasonable to enquire about the special features of a normed linear space $X$ when the $M_{\varepsilon}(X)$ sets satisfy a uniform condition.

The norm of a normed linear space $X$ is said to be uniformly strongly differentiable (uniformly Fréchet differentiable) on a set $A \subseteq S(X)$ if 
for all $x \in A$ and $y \in S(X)$,

$$
\lim _{\lambda \rightarrow 0} \frac{\|x+\lambda y\|-\|x\|}{\lambda}
$$

exists and is approached uniformly for all $x \in A$ and $y \in S(X)$; or equivalently, for any given $\varepsilon>0$, there exists a $\delta(\varepsilon)>0$ such that for all $x \in A$,

$\|x+y\|+\|x-y\|<2+\varepsilon\|y\|$ for all $y \in X$, where $\|y\|<\delta$.

Now given $\varepsilon, \delta>0$, we denote by $M_{\varepsilon, \delta}(X)$ the set of points of $S(X)$ such that

$\|x+y\|+\|x-y\|<2+\varepsilon\|y\|$ for all $y \in X$, where $\|y\|<\delta$.

The following lemma provides the mechanism for determining those spaces which satisfy conditions analogous to those studied for property $(I)$.

LEMMA 5.1. For a normed linear space $X$, suppose that for some $\varepsilon, \delta>0, D\left(M_{\varepsilon, \delta}(X)\right)$ is norm dense in $S\left(X^{*}\right)$. Then for any $2>\varepsilon_{1}>\varepsilon>0$ and $f, g \in S\left(X^{*}\right)$, where $\|f-g\|>\varepsilon_{1}$, we have $\|f+g\|<2-\left(\varepsilon_{1}-\varepsilon\right) \delta$.

Proof. Since $D\left(M_{\varepsilon, \delta}(X)\right)$ is norm dense in $S\left(X^{*}\right)$, we have for every $f \in X^{*}$,

$$
\|f\|=\sup \left\{|f(x)|: x \in M_{\varepsilon, \delta}(X)\right\}
$$

For $f, g \in S\left(X^{*}\right)$,

$$
\begin{aligned}
\|f+g\| & =\sup \left\{(f+g)(x): x \in M_{\varepsilon, \delta}(X)\right\} \\
& =\sup \left\{f(x+y)+g(x-y)-(f-g)(y): x \in M_{\varepsilon, \delta}(X)\right\} \text { for all } y \in X \\
& \leq 2+\varepsilon \delta-(f-g)(y) \text { for all } y \in X \text { such that }\|y\|<\delta .
\end{aligned}
$$

If $\|f-g\|>\varepsilon$, we can choose $y$ such that $\|y\|<\delta$ and $(f-g)(y)>\varepsilon \delta$, and then $\|f+g\|<2-\left(\varepsilon_{1}-\varepsilon\right) \delta$.

Our theorem shows the power of the assumption of our uniform condition.

THEOREM 5.1. (i) For a normed linear space $X$, suppose that for 
some $0<\varepsilon<2$ and $\delta>0, D\left(M_{E, \delta}(X)\right)$ is norm dense in $S\left(X^{*}\right)$. Then $X$ has an equivalent norm which is uniformly strongly differentiable on $S(X)$.

(ii) For a normed linear space $X$, suppose that for any given $0<\varepsilon<2$ there exists a $\delta(\varepsilon)>0$ such that $D\left(M_{\varepsilon, \delta}(X)\right)$ is norm dense in $S\left(X^{*}\right)$. Then the norm of $X$ is uniformly strongly differentiable on $S(X)$.

Proof. (i) From Lemma 5.1, $X$ is "inquadrate" which implies that there exists an equivalent norm on $X$ which is uniformly strongly differentiable on $S(X)$ [3, p. 169].

(ii) From Lemma 5.1, $X^{*}$ is uniformly rotund and so the norm of $X$ is uniformly strongly differentiable on $S(X)$.

\section{References}

[1] Béla Bollobás, "An extension to the theorem of Bishop and Phelps", Bulz. London Math. Soc. 2 (1970), 181-182.

[2] James B. Collier, "The dual of a space with the Radon-Nikodym property", Pacific J. Math. 64 (1976), 103-106.

[3] Mahlon M. Day, Normed Zinear spaces, 3rd ed. (Ergebnisse der Mathematik und ihrer Grenzgebiete, 21 . Springer-Verlag, Berlin, Heidelberg, New York, 1973).

[4] J. Diestel and J.J. UhI, Jr., "The Radon-Nikodym theorem for Banach space valued measures", Rocky Mountain J. Math. 6 (1976), 1-46.

[5] J.R. Giles, "On a characterisation of differentiability of the norm of a normed linear space", J. Austral. Math. Soc. 12 (1971), $106-114$.

[6] K. John and V. Zizler, "A renorming of dual spaces", Israel J. Math. 12 (1972), 331-336.

[7] S. Mazur, "Über schwache Konvergenz in den Räumen $\left(I^{p}\right)$ ", Studia Math. 4 (1933), 128-133. 
[8] I. Namioka and R.R. Phelps, "Banach spaces which are Asplund spaces", Duke Math. J. 42 (1975), 735-750.

[9] R.R. Phelps, "A representation theorem for bounded convex sets", Proc. Amer. Math. Soc. 11 (1960), 976-983.

[10] R.R. Phelps, "The duality between Asplund and Radon-Nikodym spaces" (Rainwater Seminar Notes, University of Washington, Seattle, 1977).

[11] V.L. Smulian, "Sur la dérivabilité de la norme dans l'espace de Banach", C.R. (DokZ.) Acad. Sci. URRS 27 (1940), 643-648.

[12] Francis Sullivan, Dentability, smoothability and stronger properties in Banach spaces", Indiana Math. J. 26 (1977), 545-553.

\footnotetext{
Department of Mathematics,

University of Newcastle,

Newcastle,

New South Wales;

Department of Mathematics,

Queen's University,

Kingston,

Ontario,

Canada ;

Department of Mathematics,

University of New England,

Armidale,

New South wales.
} 